\title{
Cranial nerve involvement in patients with leprous neuropathy
}

\author{
Sudhir Kumar, Mathew Alexander, Chandran Gnanamuthu \\ Neurology Unit, Department of Neurological Sciences, Christian Medical College, Vellore, Tamil Nadu, India
}

\begin{abstract}
Background: Leprosy is one of the most common causes of peripheral neuropathy, perhaps closely matched by diabetic neuropathy. Patterns of peripheral neuropathy in leprosy can be varied, which may include mononeuropathy, mononeuritis multiplex and symmetric polyneuropathy. Cranial nerves, especially facial and trigeminal nerves, are also commonly involved in leprosy. Aims: To find out the pattern and spectrum of cranial nerve involvement in a consecutive series of patients with leprous neuropathy. Settings and Design: A retrospective review of patients admitted with leprosy to the Neurology Department of a tertiary care center. Materials and Methods: All consecutive patients admitted during an 8-year period (1995-2003) and diagnosed to have leprosy were included. They were clinically evaluated to determine the frequency and pattern of cranial nerve involvement. Results: About $18 \%(9 / 51)$ of the leprosy patients seen during that period had clinical evidence of cranial nerve involvement. Facial and trigeminal nerves were the most commonly affected (five and four patients respectively). Conclusions: Cranial nerve involvement is common in leprosy, which emphasizes the need to carefully examine them. Also, one should exclude leprosy in patients presenting with isolated cranial neuropathies.
\end{abstract}

Key words: Cranial neuropathy, leprosy

\section{Introduction}

Leprosy is the most common cause of treatable peripheral neuropathy in India and probably also in the world. Cutaneous nerves are universally affected in leprosy, even in cases of pure neuritic leprosy with apparently normal skin. ${ }^{[1]}$ Though the skin and peripheral nerves are more commonly affected in leprosy, other sites such as eyes, upper respiratory tract, testes, hands and feet can also get affected. Leprous neuropathy is characterized by the involvement of superficial peripheral nerve trunks in cooler body regions such as ulnar, median, radial, common peroneal, supraorbital and great auricular nerves. ${ }^{[2]}$ Leprous neuropathy can take any of the three forms: mononeuropathy, mononeuritis multiplex or symmetric polyneuropathy. Cranial nerve involvement is also commonly seen in patients with leprosy. Most of this information is derived from isolated case reports that highlight the involvement of facial, trigeminal or other nerves. ${ }^{[3-7]}$ There are other reports highlighting involvement of multiple cranial nerves (polyneuritis cranialis). ${ }^{[8-10]}$ There is a paucity of larger studies on this topic. ${ }^{[11,12]}$ The present study highlights the pattern of cranial nerve involvement seen in patients with leprous neuropathy in a hospital in South India.

\section{Materials and Methods}

The study was conducted in a tertiary care hospital in South India during an 8-year period (January 1995 to January 2003). All patients presenting with peripheral neuropathy were evaluated for leprosy. A detailed clinical examination was done to look for hypopigmented anesthetic patches, nerve thickening and other stigmata of leprosy, such as madarosis, infiltration of earlobes, resorption of toes and fingers. Dermatologists also examined the patients and skin smears were taken to look for the presence of acid-fast bacilli. Nerve conduction studies were done in majority of the cases. A nerve biopsy was done, if required, for confirming the diagnosis of leprosy. All the patients who were diagnosed to have peripheral neuropathy due to leprosy were included in the study. Cranial nerves ( $3^{\text {rl }}$ to $12^{\text {th }}$ ) were clinically examined for any evidence of involvement and the pattern was noted.

\section{Results}

A total of 51 patients (all males) with leprous neuropathy were seen during the study period. The mean age was 54 years (range $20-76$ years). Nine out of these $(17.6 \%)$ had clinical evidence of cranial nerve involvement. Six of the patients were from Tamil $\mathrm{Nadu}$, two from Bihar and one from Nepal (reflecting the referral patterns of the hospital). 


\section{Type of leprosy}

Five had lepromatous type, two had tuberculoid type and one each had mid-borderline and pure neuritic form of the disease.

\section{Duration of leprosy}

The duration between the diagnosis of leprosy and onset of cranial nerve symptoms ranged from 4 months to 13 years (mean: 3.7 years).

\section{Pattern of cranial nerve involvement}

The pattern of cranial nerve involvement is summarized in Table 1. The most common cranial nerve affected was the facial (involved in five patients). Trigeminal nerve was affected in four and the glossopharyngeal nerve in two patients. Oculomotor, auditory, vagus, spinal accessory and hypoglosal nerves were affected in one patient each. Four (44\%) patients had multiple cranial nerve involvement. Bilateral cranial nerve involvement was seen in five patients. Loss of taste over the anterior two-thirds of the tongue was seen in two patients and in one of them, it was the only feature of facial nerve involvement present.

\section{Presence of associated peripheral neuropathy}

All patients had clinical or electrophysiological or both evidence of peripheral neuropathy. Nerve conduction studies showed features of symmetrical axonal and demyelinating sensorimotor neuropathy in four patients; mononeuritis multiplex (asymmetric sensorimotor axonal neuropathy) in three and mononeuropathy in two patients (left radial and left ulnar neuropathy in one each).

\section{Nerve biopsy findings}

Nerve biopsy was performed in 17 patients. Features of leprosy were seen in all cases. This included lepromatous in 8, tuberculoid in 7 and mid-borderline in 2 patients. All patients with cranial neuropathy underwent nerve biopsy and the number of patients in the above categories included 5,3 and 1 patient(s) respectively.

\section{Presence of other features of leprosy}

Skin lesions suggestive of leprosy (hypopigmented anesthetic macules) were present in six patients. Of the remaining three, one had madarosis and resorption of toes and another had trophic ulcers and resorption of toes. Only one patient had pure neuritic type of leprosy.

\section{Discussion}

All patients in our series were males. Leprosy is known to affect men more commonly than women. This could be related to better immune status in women, lesser opportunities to contract the disease as they predominantly remain homebound and lesser opportunities to seek health care.

Our study showed that $18 \%$ of patients with leprous neuropathy admitted to a Neurology ward in a tertiary care hospital had clinical evidence of cranial nerve involvement. However, extension of this study till recently had shown a prevalence of $16.5 \%$ (15/ 91 patients). ${ }^{[11]}$ Previous studies have also reported a high prevalence of cranial nerve involvement. ${ }^{[3,4,12]}$ The data is summarized in Table 2.

Facial nerve involvement in leprosy is reported to occur in about $10 \%$ of the patients and is the most common nerve affected. Our findings in this regard are similar to another Indian study. ${ }^{[12}$ Lagophthalmos and corneal xerosis/ulceration are the likely complications, especially in patients with impaired corneal sensations, which is also common in leprosy. It has been found that leprosy affects facial nerve in a scattered distribution from the main trunk to all peripheral branches ${ }^{[5]}$ and is not confined to peripheral zygomatic branch, as was thought earlier. In another study, ${ }^{[6]}$ it was found that the involvement of the facial nerve occurred late in the disease and had an average duration of 12.1 years. However, in a recent Indian study, the duration of disease was less than 5 years in all patients with cranial neuropathy (a finding similar to our study). ${ }^{[12]}$

\begin{tabular}{ccccc}
\hline \multicolumn{4}{c}{ Table 1: Pattern of cranial and peripheral nerve involvement in study patients } \\
\hline Age & Disease duration & Type of leprosy & Cranial nerves affected & Pattern of neuropathy \\
53 & 1 year & BL & Bilateral $5,7,9$ and 10 & Mononeuritis multiplex \\
41 & 6 years & B & Bilateral 5 and 9 & Mononeuritis multiplex \\
57 & 4 months & Right 5 and 7 & Left radial neuropathy \\
50 & 13 years & LL & Right 3, 7 and 11 & Symmetric polyneuropathy \\
76 & 4 years & LL & Bilateral 8 & Symmetric polyneuropathy \\
54 & 8 months & Bilateral 5 & Mononeuritis multiplex \\
20 & 2 years & L & Left 7 & Left ulnar neuropathy \\
67 & 3 years & LL & Bilateral 7 & Symmetric polyneuropathy \\
65 & 3 years & LL & Left 12 & Symmetric polyneuropathy \\
\hline
\end{tabular}

LL-Lepromatous; TT-Tuberculoid; BB-Mid-borderline type of leprosy

Table 2: Comparison of cranial nerve involvement in various studies

Authors (year)

Mann SB et al (1987)[3]

Koyuncu et al (1994) ${ }^{[4]}$

Gopinath et al (2004) ${ }^{[12]}$

Kumar S et al (present)

$\begin{array}{cc}\text { Total number } & \text { Trigeminal (\%) } \\ 25 & \text { Not studied } \\ 39 & 2(5.5) \\ 100 & 7(7) \\ 51 & 4(8)\end{array}$

51
Pattern of neuropathy

ononeuritis multiplex Mononeuritis multiplex Left radial neuropathy Symmetric polyneuropathy Symmetric polyneuropathy 
Trigeminal nerve is also frequently involved. It seems to be the second most common cranial nerve to get affected in leprosy. In an earlier study, hypesthesia and anesthesia were most often observed in the maxillary divisions of the trigeminal nerve. ${ }^{[6]}$

Vestibulocochlear nerve function was assessed by Mann et al. ${ }^{[3]}$ Out of 25 bacillary positive cases evaluated, 4 patients complained of impaired hearing. On testing, cochlear type of hearing impairment was seen in 11 patients (44\%). Similarly, Koyuncu et $a l^{[4]}$ found cochlear nerve involvement in $22 \%$ of cases studied. This is significantly more than what we found (1 patient, $2 \%$ of cases). However, it should be noted that audiometric testing was not performed in our patients, which could be a limitation in assessing the exact involvement of auditory nerve.

Loss of taste was seen in 2 of our cases. Taste was evaluated in an earlier study among 30 patients. ${ }^{[7]}$ Twelve (40\%) of these patients had evidence of some degree of taste impairment. It was related to the severity of the disease.

Our study focused on determining the cranial nerve involvement in patients already diagnosed to have leprous neuropathy. However, it is important to note that cranial neuropathy can be the presenting feature of leprosy. ${ }^{[11]}$ Reported presenting features have been trigeminal neuralgia, facial and hypoglossal palsy.

Multiple cranial nerve involvement (polyneuritis cranialis) was seen in four out of nine (44\%) patients in our study. Polyneuritis cranialis has been earlier described in three separate case reports. ${ }^{[8-}$ ${ }^{10]}$ Dhar et al were the first to emphasize the involvement of glossopharyngeal, vagus and hypoglossal nerves in a case of lepromatous leprosy. ${ }^{[10]}$ Dhar et al reported the involvement of trigeminal, abducens, facial and cochlear nerves in an 80-year old patient with borderline lepromatous leprosy. ${ }^{[8]}$

In summary, cranial nerves are commonly involved in patients with leprosy. Though the involvement tends to be more common in patients with lepromatous type of leprosy of longer duration, it can occur in other forms of leprosy and short-duration disease too. Associated features of leprosy such as madarosis, resorption of digits and toes and trophic ulcers are seen in majority of cases with leprous cranial neuropathy.

\section{Conclusions}

One should thoroughly examine the cranial nerve functions in a case of leprosy as this disease commonly affects cranial nerves. Similarly, one should look for any evidence of leprosy in a patient presenting with unexplained cranial nerve symptoms.

\section{References}

1. Suneetha S, Arunthathi S, Chandi S, Kurian N, Chacko C.J. Histological studies in primary neuritic leprosy: Changes in the apparently normal skin. Lepr Rev 1998;69:351-7.

2. Nations SP, Katz JS, Lyde CB, Barohn RJ. Leprous neuropathy: An American perspective. Semin Neurol 1998;18:113-24.

3. Mann SB, Kumar B, Yande R, Kaur S, Kaur I, Mehra YN. Eighth nerve evaluation in leprosy. Indian J Lepr 1987;59:20-5.

4. Koyuncu M, Celik O, Ozturk A, Saunders M. Audiovestibular system, fifth and seventh cranial nerve involvement in leprosy. Indian J Lepr 1994;66:421-8.

5. Turkoff E, Richard B, Assadian O, Khatri B, Knolle E, Lucas S. Leprosy affects facial nerves in a scattered distribution from the main trunk to all peripheral branches and neurolysis improves muscle function of the face. Am .J Trop Med Hyg 2003;68:81-8

6. Reichart PA, Srisuwan S, Metah D. Lesions of the facial and trigeminal nerve in leprosy. An evaluation of 43 cases. Int J Oral Surg 1982;11:14-20.

7. Soni NK, Chatterji P. Disturbance of taste in leprosy. J Laryngol Otol 1981;95:71720.

8. Dhar MC, Ghosh KC, Basu K, Banerjee G. Polyneuritis cranialis in a case of leprosy. J Assoc Physic India 1999;47:828-30.

9. Paul JT, North-Wilhelm K, Higdon GA, Chesser RS, Hayden DL. Multiple cranial neuropathies associated with leprosy. South Med J 1994;87:937-40.

10. Dhar S, Sharma VK, Kaur S. Facial, glossopharyngeal, vagus and hypoglossal nerve palsy in a case of lepromatous leprosy. Indian J Lepr 1993;65:333-6.

11. Kumar S. Cranial nerve involvement in leprosy. Indian J Lepr 2005;77:177-8.

12. Gopinath DV, Thapa DM, Jaishankar T.J. A clinical study of the involvement of cranial nerves in leprosy. Indian .J Lepr 2004;76:1-9.

Accepted on 10-05-2006 